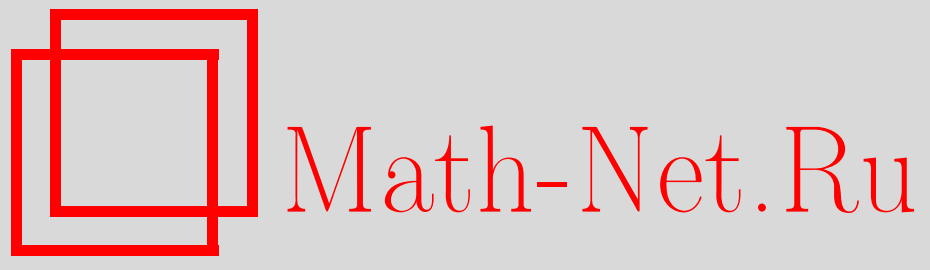

С. А. Хихол, Усреднение локальных характеристик сближает семимартингал с независимыми приращениями с процессами Леви, Теория вероятн. и ее примен., 2013, том 58, выпуск $3,486-505$

DOI: https://doi.org/10.4213/tvp4522

Использование Общероссийского математического портала Math-Net.Ru подразумевает, что вы прочитали и согласны с пользовательским соглашением http: //www . mathnet.ru/rus/agreement

Параметры загрузки:

IP: 54.196 .121 .252

26 апреля 2023 г., 10:25:06

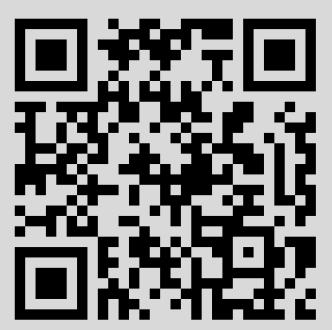




\title{
УСРЕДНЕНИЕ ЛОКАЛЬНЫХ ХАРАКТЕРИСТИК СБЛИЖАЕТ СЕМИМАРТИНГАЛ С НЕЗАВИСИМЫМИ ПРИРАЩЕНИЯМИ С ПРОЦЕССАМИ ЛЕВИ
}

\begin{abstract}
Усреднение локальных характеристик по времени преобразует семимартингал с независимыми приращениями в процесс Леви. Показано, что этот процесс Леви «ближе» к любому процессу Леви, чем исходный процесс, где «бо́льшая близость» процессов понимается как бо́льшая близость всех $f$-дивергенций между их распределениями, что также допускает эквивалентную формулировку в терминах сравнения соответствующих бинарных статистических экспериментов. Кроме того, доказан критерий эквивалентности бинарных экспериментов, составленных из распределения семимартингала с независимыми приращениями и распределения процесса Леви.

Ключевые слова и фразы: семимартингал, процесс с независимыми приращениями, процесс Леви, бинарный статистический эксперимент, $f$-дивергенция, процесс плотности, триплет локальных характеристик.
\end{abstract}

1. Введение. Термины и обозначения, не объясняемые ниже, заимствованы из монографии Ж. Жакода и А. Н. Ширяева [12].

Пусть $\Omega=\mathbb{D}\left(\mathbf{R}^{d}\right)=\mathbb{D}\left([0,1] ; \mathbf{R}^{d}\right)$ - пространство всех непрерывных справа и имеющих пределы слева функций $\omega:[0,1] \rightarrow \mathbf{R}^{d}, X-$ канонический процесс, задаваемый соотношением $X_{t}(\omega)=\omega(t)$, фильтрация $\mathbb{F}=\left(\mathscr{F}_{t}\right)_{t \in[0,1]}$ порождена процессом $X$, т.е. $\mathscr{F}_{t}=\bigcup_{\varepsilon>0} \mathscr{F}_{t+\varepsilon}^{0}$, $\mathscr{F}_{t}^{0}=\sigma\left\{X_{s}, s \leqslant t\right\}, \mathscr{F}=\mathscr{F}_{1}$.

Введем обозначения для некоторых классов вероятностных мер на $(\Omega, \mathscr{F})$. Класс $\mathscr{P}_{I}$ состоит из всех вероятностных мер, по которым процесс $X$ является семимартингалом с независимыми приращениями, т.е. $X$ - семимартингал и для любых $0 \leqslant s \leqslant t$ случайная величина $X_{t}-X_{s}$ не зависит от $\sigma$-алгебры $\mathscr{F}_{s}$ (в отличие от монографии [12], мы не предполагаем, что $X_{0}=0$ п.н.). Определим также

$$
\begin{aligned}
\mathscr{P}_{L} & =\left\{\mathrm{P} \in \mathscr{P}_{I}: X-X_{0} \text { есть процесс Леви относительно } \mathrm{P}\right\} \\
\mathscr{P}_{L, 0} & =\left\{\mathrm{P} \in \mathscr{P}_{L}: \mathrm{P}\left(X_{0}=0\right)=1\right\} .
\end{aligned}
$$

* Московский государственный университет им. М. В. Ломоносова, механико-математический факультет, Москва, Россия; e-mail: sahihol@gmail.com 
Разумеется, введенные классы зависят от размерности $d$ канонического процесса. Хотя в дальнейшем мы будем иметь дело с распределениями процессов различной размерности, мы предпочитаем не перегружать обозначения указанием размерности. Если меры отвечают распределениям процессов размерности, отличной от $d$, это будет оговариваться отдельно.

Основной результат статьи состоит в следующем. Пусть $\mathrm{P}^{\prime} \in \mathscr{P}_{I}$, тогда найдется такая мера $\overline{\mathrm{P}} \in \mathscr{P}_{L}$, что для любой $\mathrm{P} \in \mathscr{P}_{L, 0}$ бинарный статистический эксперимент $(\Omega, \mathscr{F},(\mathrm{P}, \overline{\mathrm{P}}))$ является менее информативным, чем $\left(\Omega, \mathscr{F},\left(\mathrm{P}, \mathrm{P}^{\prime}\right)\right)$. Иными словами, для любой $\mathrm{P} \in \mathscr{P}_{L, 0}$ и для любой выпуклой функции $f:(0, \infty) \rightarrow \mathbf{R}$ имеет место следующее неравенство для $f$-дивергенций:

$$
\mathscr{J}_{f}(\overline{\mathrm{P}}, \mathrm{P}) \leqslant \mathscr{J}_{f}\left(\mathrm{P}^{\prime}, \mathrm{P}\right)
$$

Детальное изложение теории статистических экспериментов имеется в монографиях [17], [21]-[23], теории $f$-дивергенций — в монографии [18]; всю необходимую для целей настоящей статьи информацию можно найти в статье [1]. Отметим также, что мера $\bar{P}$ строится явно по мере $\mathrm{P}^{\prime}$ по сути дела усреднением по времени локальных характеристик процесса $X$ (см. (2) ниже).

О связи полученного результата с результатами ряда работ (среди которых наиболее близкими являются [8], [10] и [14]), мотивированных приложениями к финансовой математике, см. в п. 4.

Итак, пусть на $(\Omega, \mathscr{F})$ задана вероятностная мера $\mathrm{P}^{\prime} \in \mathscr{P}_{I}$. В дальнейшем фиксируем произвольную функцию усечения $h: \mathbf{R}^{d} \rightarrow \mathbf{R}^{d}$. Хорошо известно, что триплет характеристик процесса $X$ относительно меры $\mathrm{P}^{\prime}$ можно выбрать детерминированным. Обозначим этот триплет $\mathbb{T}^{\mathrm{P}^{\prime}}$ :

$$
\mathbb{T}^{\mathrm{P}^{\prime}}=\left(B_{t}^{\prime}, C_{t}^{\prime}, \nu^{\prime}(d t, d x)\right)
$$

Рассмотрим также триплет

$$
\mathbb{T}^{\overline{\mathrm{P}}}=(\bar{b} t, \bar{c} t, d t \bar{F}(d x)),
$$

где

$$
\bar{b}=B_{1}^{\prime}, \quad \bar{c}=C_{1}^{\prime}, \quad \bar{F}(A)=\nu^{\prime}([0,1] \times A), \quad A \in \mathscr{B}\left(\mathbf{R}^{d}\right) .
$$

Введем $\overline{\mathrm{P}}$ как такую меру из $\mathscr{P}_{L}$, что $\mathscr{L}$ aw $\left(X_{0} \mid \overline{\mathrm{P}}\right)=\mathscr{L}$ aw $\left(X_{0} \mid \mathrm{P}^{\prime}\right)$ и триплет процесса $X$ относительно $\overline{\mathrm{P}}$ есть $\mathbb{T}^{\overline{\mathrm{P}}}$. Поскольку

$$
\int_{\mathbf{R}^{d}}\left(|x|^{2} \wedge 1\right) \bar{F}(d x)=\int_{\mathbf{R}^{d}} \int_{0}^{1}\left(|x|^{2} \wedge 1\right) \nu^{\prime}(d t, d x)<\infty,
$$


существование такой меры $\overline{\mathrm{P}}$ следует из детерминированности и однородности по времени триплета $\mathbb{T}^{\bar{P}}$ (см., например, $[12$, следствие II.14.19]), а ее единственность очевидна.

Заметим, что если $X$ является локальным мартингалом (и, следовательно, мартингалом, см., например, [3, теорема 5.8]) по мере $\mathrm{P}^{\prime}$, то он является мартингалом и по мере $\bar{P}$. Это вытекает из $[12$, предложение II.2.29] в силу следующих соотношений:

$$
\begin{aligned}
\int_{\mathbf{R}^{d}}\left(|x|^{2} \wedge|x|\right) \bar{F}(d x) & =\int_{\mathbf{R}^{d}} \int_{0}^{1}\left(|x|^{2} \wedge|x|\right) \nu^{\prime}(d t, d x)<\infty \\
\int_{\mathbf{R}^{d}}(h(x)-x) \bar{F}(d x) & =\int_{\mathbf{R}^{d}} \int_{0}^{1}(h(x)-x) \nu^{\prime}(d t, d x)=B_{1}^{\prime}=\bar{b} .
\end{aligned}
$$

Отметим также, что если $X$ является $\sigma$-мартингалом, но не локальным мартингалом по мере $\mathrm{P}^{\prime}$, то $X$ не будет $\sigma$-мартингалом по мере $\overline{\mathrm{P}}$.

Заметим, что если $X$ не имеет фиксированных моментов разрыва относительно $\mathrm{P}^{\prime}$, то мера $\overline{\mathrm{P}}$ описывается совсем просто: это такая мера из $\mathscr{P}_{L}$, что $\mathscr{L}$ aw $\left(\left(X_{0}, X_{1}\right) \mid \overline{\mathrm{P}}\right)=\mathscr{L}$ aw $\left(\left(X_{0}, X_{1}\right) \mid \mathrm{P}^{\prime}\right)$. Это вытекает из теоремы II.4.15 монографии [12].

Теорема 1. Для любой мерь $\mathrm{P} \in \mathscr{P}_{L, 0}$ и введенных выше мер $\mathrm{P}^{\prime}, \overline{\mathrm{P}}$ статистический эксперимент $(\mathrm{P}, \overline{\mathrm{P}})$ менее информативен, чем $\left(\mathrm{P}, \mathrm{P}^{\prime}\right)$.

3 а м е ч ан и е 1 . В действительности в доказательстве теоремы 1 используется не совпадение распределений $X_{0}$ по $\overline{\mathrm{P}}$ и $\mathrm{P}^{\prime}$, а только равенство $\overline{\mathrm{P}}\left(X_{0}=0\right)=\mathrm{P}^{\prime}\left(X_{0}=0\right)$. Более того, утверждение теоремы 1 сохранится, если меру $\overline{\mathrm{P}}$ заменить на такую меру $\overline{\mathrm{Q}} \in \mathscr{P}_{L}$, что триплет $X$ относительно $\overline{\mathrm{Q}}$ есть по-прежнему $\mathbb{T}^{\overline{\mathrm{P}}}$ и $\overline{\mathrm{Q}}\left(X_{0}=0\right) \geqslant \mathrm{P}^{\prime}\left(X_{0}=0\right)$ : как легко видеть из приводимой ниже формулы $(5)$, в этом случае эксперимент $(P, \bar{Q})$ менее информативен, чем $(P, \bar{P})$. В частности, в качестве $\overline{\mathrm{Q}}$ можно взять такую меру из $\mathscr{P}_{L, 0}$, что $X$ является процессом Леви с триплетом $\mathbb{T}^{\overline{\mathrm{P}}}$.

Первая идея доказательства теоремы 1 состоит в том, чтобы использовать так называемую лемму о сравнении из статьи [1] (лемма 5.1). Однако условия леммы о сравнении являются только достаточными для большей/меньшей информативности бинарных экспериментов и не всегда выполняются в условиях теоремы 1.

Тем не менее с помощью леммы о сравнении (а точнее, ее частного случая, см. [1, раздел 5.4]) оказывается возможным доказать, что эксперимент $(P, \bar{P})$ менее информативен, чем некоторый эксперимент $\left(Q, Q^{\prime}\right)$, который, в свою очередь, эквивалентен $\left(\mathrm{P}, \mathrm{P}^{\prime}\right)$ ! Более того, меры $\mathrm{Q}$ и $\mathrm{Q}^{\prime}$ можно выбрать из классов $\mathscr{P}_{L, 0}$ и $\mathscr{P}_{L}$ соответственно на пространстве $\mathbb{D}(\mathbf{R})$.

Теорема 1 доказывается в п. 3. При этом для доказательства эквивалентности экспериментов $\left(\mathrm{P}, \mathrm{P}^{\prime}\right)$ и $\left(\mathrm{Q}, \mathrm{Q}^{\prime}\right)$ используется результат, дока- 
зываемый в п. 2 двумя различными способами и имеющий самостоятельный интерес, а именно, приводятся необходимые и достаточные условия эквивалентности экспериментов $\left(\mathrm{P}, \mathrm{P}^{\prime}\right)$ и $\left(\mathrm{Q}, \mathrm{Q}^{\prime}\right)$, где, как и выше, меры $\mathrm{P} \in \mathscr{P}_{L, 0}, \mathrm{P}^{\prime} \in \mathscr{P}_{I}$ заданы на $\mathbb{D}\left(\mathbf{R}^{d}\right)$, а меры $\mathrm{Q} \in \mathscr{P}_{L, 0}, \mathrm{Q}^{\prime} \in \mathscr{P}_{I}$ заданы на $\mathbb{D}\left(\mathbf{R}^{d^{\prime}}\right)$.

Некоторые вспомогательные результаты вынесены в приложение.

2. Критерий эквивалентности экспериментов. В этом пункте фиксированы вероятностные меры $\mathrm{P} \in \mathscr{P}_{L, 0}, \mathrm{P}^{\prime} \in \mathscr{P}_{I}$ на $\mathbb{D}\left(\mathbf{R}^{d}\right)$ и $\mathrm{Q} \in \mathscr{P}_{L, 0}, \mathrm{Q}^{\prime} \in \mathscr{P}_{I}$ на $\mathbb{D}\left(\mathbf{R}^{d^{\prime}}\right)$. Наша цель - установить критерий эквивалентности экспериментов $\left(\mathrm{P}, \mathrm{P}^{\prime}\right)$ и $\left(\mathrm{Q}, \mathrm{Q}^{\prime}\right)$.

Выберем детерминированные версии триплетов канонических процессов по мерам $\mathrm{P}, \mathrm{P}^{\prime}, \mathrm{Q}, \mathrm{Q}^{\prime}$ и обозначим их

$$
\begin{gathered}
\mathbb{T}^{\mathrm{P}}=(b t, c t, d t F(d x)), \quad \mathbb{T}^{\mathrm{P}^{\prime}}=\left(B_{t}^{\prime}, C_{t}^{\prime}, \nu^{\prime}(d t, d x)\right), \\
\mathbb{T}^{\mathrm{Q}}=\left(b_{\mathrm{Q}} t, c_{\mathrm{Q}} t, d t G(d x)\right), \quad \mathbb{T}^{\mathrm{Q}^{\prime}}=\left(B_{\mathrm{Q}, t}^{\prime}, C_{\mathrm{Q}, t}^{\prime}, \nu_{\mathrm{Q}}^{\prime}(d t, d x)\right) .
\end{gathered}
$$

Обозначим также $\nu(d t, d x)=d t F(d x), \nu_{\mathrm{Q}}(d t, d x)=d t G(d x)$.

Вопрос об эквивалентности экспериментов решается просто, если в одной из пар $\left(\mathrm{P}, \mathrm{P}^{\prime}\right)$ или $\left(\mathrm{Q}, \mathrm{Q}^{\prime}\right)$ меры сингулярны. Действительно, в этом случае эксперимент, отвечающий этой паре, является вполне информативным и эквивалентен другому эксперименту тогда и только тогда, когда и другой эксперимент является вполне информативным, т.е. и в другой паре меры сингулярны. Поскольку условия сингулярности рассматриваемых пар мер хорошо известны (см. теорему 4 в приложении), в дальнейшем мы предполагаем, что меры $\mathrm{P}$ и $\mathrm{P}^{\prime}$, а также $\mathrm{Q}$ и $\mathrm{Q}^{\prime}$ не сингулярны. В частности, $C_{t}^{\prime}=c t$ и $C_{\mathrm{Q}, t}^{\prime}=c_{\mathrm{Q}} t$ (см. теорему 4$)$.

Введем для пары мер $\mathrm{P}$ и $\mathrm{P}^{\prime}$ несколько дополнительных объектов.

Пусть $\mu$ - мера скачков $d$-мерного канонического процесса $X$. Для произвольной меры $\mathrm{R}$ обозначим $\mathrm{R}_{0}=\mathrm{R}\left(X_{0}=0\right)$.

Пусть $\lambda$ - мера на $\mathscr{B}\left([0,1] \times \mathbf{R}^{d}\right)$ такая, что $\left(|x|^{2} \wedge 1\right) * \lambda_{1}<\infty$, $\nu \ll \lambda$ и $\nu^{\prime} \ll \lambda$. Положим $U=d \nu / d \lambda, U^{\prime}=d \nu^{\prime} / d \lambda, A=\{U>0\}$, $Y=\left(U^{\prime} / U\right) 1_{A}+1_{A^{c}}, \nu_{1}^{\prime}=1_{A} \cdot \nu^{\prime}, \nu_{2}^{\prime}=1_{A^{c}} \cdot \nu^{\prime}$ и $a_{t}^{\prime}=\nu^{\prime}\left(\{t\} \times \mathbf{R}^{d}\right)$. Заметим, что $\nu_{1}^{\prime} \ll \nu, \nu_{1}^{\prime}=Y \cdot \nu$ и $\nu_{2}^{\prime} \perp \nu$.

По теореме 4 существует измеримая функция $\beta:[0,1] \rightarrow \mathbf{R}^{d}$ такая, что

$$
B_{t}^{\prime}=B_{t}+h(x)\left(U^{\prime}-U\right) * \lambda_{t}+\int_{0}^{t} c \beta_{s} d s
$$

Аналогичные объекты для пары мер $\mathrm{Q}, \mathrm{Q}^{\prime}$ обозначим $\beta^{\mathrm{Q}}, Y^{\mathrm{Q}}, \nu_{\mathrm{Q}, 2}^{\prime}, a_{\mathrm{Q}}^{\prime}$.

Теорема 2 (критерий эквивалентности). Эксперименты $\left(\mathrm{P}, \mathrm{P}^{\prime}\right) u$ $\left(\mathrm{Q}, \mathrm{Q}^{\prime}\right)$ эквивалентнь тогда и только тогда, когда вьлолняется 
условие

$$
\left\{\begin{array}{l}
\int_{0}^{1} \beta_{s}^{\top} c \beta_{s} d s=\int_{0}^{1}\left(\beta_{s}^{\mathrm{Q}}\right)^{\top} c_{\mathrm{Q}} \beta_{s}^{\mathrm{Q}} d s, \\
\nu(Y \in B)=\nu^{\mathrm{Q}}\left(Y^{\mathrm{Q}} \in B\right), \quad B \in \mathscr{B}\left(\mathbf{R}_{+} \backslash\{1\}\right), \\
\frac{\mathrm{P}_{0}^{\prime} \prod_{0<s \leqslant 1}\left(1-a_{s}^{\prime}\right)}{\exp \left(\nu_{2}^{\prime}\left\{(s, x): 0<s \leqslant 1, a_{s}^{\prime}=0\right\}\right)}=\frac{\mathrm{Q}_{0}^{\prime} \prod_{0<s \leqslant 1}\left(1-a_{\mathrm{Q}, s}^{\prime}\right)}{\exp \left(\nu_{\mathrm{Q}, 2}^{\prime}\left\{(s, x): 0<s \leqslant 1, a_{\mathrm{Q}, s}^{\prime}=0\right\}\right)} .
\end{array}\right.
$$

Следствие 1. Пусть $\mathrm{P}, \mathrm{P}^{\prime} \in \mathscr{P}_{L, 0}$ заданьл на $\mathbb{D}\left(\mathbf{R}^{d}\right) u \mathrm{Q}, \mathrm{Q}^{\prime} \in \mathscr{P}_{L, 0}$ заданьл на $\mathbb{D}\left(\mathbf{R}^{d^{\prime}}\right)$. В этом случае экспериментьл $\left(\mathrm{P}, \mathrm{P}^{\prime}\right)$ u $\left(\mathrm{Q}, \mathrm{Q}^{\prime}\right)$ эквивалентны тогда и только тогда, когда выполняется условие

$$
\left\{\begin{array}{l}
\beta^{\top} c \beta=\left(\beta^{\mathrm{Q}}\right)^{\top} c_{\mathrm{Q}} \beta^{\mathrm{Q}}, \\
\nu(Y \in B)=\nu^{\mathrm{Q}}\left(Y^{\mathrm{Q}} \in B\right), \quad B \in \mathscr{B}\left(\mathbf{R}_{+} \backslash\{1\}\right), \\
\nu_{2}^{\prime}\left\{[0,1] \times \mathbf{R}^{d}\right\}=\nu_{\mathrm{Q}, 2}^{\prime}\left\{[0,1] \times \mathbf{R}^{d^{\prime}}\right\}
\end{array}\right.
$$

Д о к а з а т ель с т в о т е о р е м ы 2 (первый способ). Покажем, что условия (Е) эквивалентны равенству интегралов Хеллингера в терминальный момент 1 для пар мер $\left(\mathrm{P}, \mathrm{P}^{\prime}\right)$ и $\left(\mathrm{Q}, \mathrm{Q}^{\prime}\right)$, т.е. $H\left(\alpha ; \mathrm{P}, \mathrm{P}^{\prime}\right)=$ $H\left(\alpha ; \mathrm{Q}, \mathrm{Q}^{\prime}\right), 0<\alpha<1$.

С этой целью выразим интеграл Хеллингера $H(\alpha)_{t}:=H\left(\alpha ; \mathrm{P}_{t}, \mathrm{P}_{t}^{\prime}\right)$ через триплеты. Известно (см. [19, теорема 1] и [12, теорема IV.4.28]), что $H(\alpha)_{t}=H(\alpha)_{0} \mathscr{E}\left(-h^{0}(\alpha)\right)_{t}$, где

$$
\begin{aligned}
h^{0}(\alpha) & =\frac{1}{2} \alpha(1-\alpha) \int \beta_{s}^{\top} c \beta_{s} d s+\varphi_{\alpha}\left(U, U^{\prime}\right) * \lambda+\sum_{s \leqslant \cdot} \varphi_{\alpha}\left(1,1-a_{s}^{\prime}\right), \\
\varphi_{\alpha}(u, v) & =\alpha u+(1-\alpha) v-u^{\alpha} v^{1-\alpha}
\end{aligned}
$$

(мы учли, что $\nu\left(\{s\} \times \mathbf{R}^{d}\right)=0$ для всех $\left.s\right)$. С учетом этого же соотношения получаем, что

$$
\begin{aligned}
\varphi_{\alpha}\left(U, U^{\prime}\right) * \lambda= & 1_{A}\left(\alpha U+(1-\alpha) U^{\prime}-U^{\alpha}\left(U^{\prime}\right)^{1-\alpha}\right) * \lambda \\
& +1_{A^{c}}(1-\alpha) U^{\prime} * \lambda \\
= & \left(\alpha+(1-\alpha) Y-Y^{1-\alpha}\right) * \nu+(1-\alpha) * \nu_{2}^{\prime}, \\
\Delta\left(\varphi_{\alpha}\left(U, U^{\prime}\right) * \lambda\right)_{s}= & (1-\alpha) a_{s}^{\prime}, \\
\varphi_{\alpha}\left(1,1-a_{s}^{\prime}\right)= & \alpha+(1-\alpha)\left(1-a_{s}^{\prime}\right)-\left(1-a_{s}^{\prime}\right)^{1-\alpha}, \\
\Delta h^{0}(\alpha)_{s}= & 1-\left(1-a_{s}^{\prime}\right)^{1-\alpha}, \\
h^{0}(\alpha)_{t}-\sum_{s \leqslant t} \Delta h^{0}(\alpha)_{s}= & \frac{1}{2} \alpha(1-\alpha) \int_{0}^{1} \beta_{s}^{\top} c \beta_{s} d s \\
& +\left(\alpha+(1-\alpha) Y-Y^{1-\alpha}\right) * \nu_{t} \\
& +(1-\alpha) \nu_{2}^{\prime}\left\{(0, t] \backslash\left\{a_{s}^{\prime}>0\right\}\right\},
\end{aligned}
$$


и, значит,

$$
\begin{aligned}
\mathscr{E}\left(-h^{0}(\alpha)\right)_{1}= & \exp \left\{-\frac{1}{2} \alpha(1-\alpha) \int_{0}^{1} \beta_{s}^{\top} c \beta_{s} d s\right\} \\
& \times \exp \left\{-\left(\alpha+(1-\alpha) Y-Y^{1-\alpha}\right) * \nu_{1}\right\} \\
& \times \exp \left\{-(1-\alpha) \nu_{2}^{\prime}\left\{(0,1] \backslash\left\{a_{s}^{\prime}>0\right\}\right\}\right\} \prod_{s \leqslant 1}\left(1-a_{s}^{\prime}\right)^{1-\alpha} .
\end{aligned}
$$

Поскольку $H(\alpha)_{0}=\left(\mathrm{P}_{0}^{\prime}\right)^{1-\alpha}$, в итоге получаем, что

$$
\begin{aligned}
H(\alpha)_{1}= & \left(\frac{\mathrm{P}_{0}^{\prime} \prod_{s \leqslant 1}\left(1-a_{s}^{\prime}\right)}{\exp \left\{\nu_{2}^{\prime}\left((0,1] \backslash\left\{a_{s}^{\prime}>0\right\}\right)\right\}}\right)^{1-\alpha} \\
& \times \exp \left\{-\frac{1}{2} \alpha(1-\alpha) \int_{0}^{1} \beta_{s}^{\top} c \beta_{s} d s\right\} \\
& \times \exp \left\{-\left(1+(1-\alpha)(Y-1)-Y^{1-\alpha}\right) * \nu_{1}\right\} \\
= & \exp \left\{(1-\alpha) C_{1}-\frac{1}{2} \alpha(1-\alpha) C_{2}-g(\alpha, x) * \widetilde{\nu}_{1}\right\},
\end{aligned}
$$

где

$$
\begin{aligned}
C_{1} & =\ln \left(\frac{\mathrm{P}_{0}^{\prime} \prod_{s \leqslant 1}\left(1-a_{s}^{\prime}\right)}{\exp \left\{\nu_{2}^{\prime}\left((0,1] \backslash\left\{a_{s}^{\prime}>0\right\}\right)\right\}}\right), \\
C_{2} & =\int_{0}^{1} \beta_{s}^{\top} c \beta_{s} d s, \\
g(\alpha, x) & =\varphi_{\alpha}(1,1+x)=1+(1-\alpha) x-(1+x)^{1-\alpha}, \\
\widetilde{\nu}(B) & =\nu(Y-1 \in B \backslash\{0\}) .
\end{aligned}
$$

Теперь очевидно, что из условия (Е) вытекает совпадение интегралов Хеллингера: $H\left(\alpha ; \mathrm{P}, \mathrm{P}^{\prime}\right)=H\left(\alpha ; \mathrm{Q}, \mathrm{Q}^{\prime}\right), 0<\alpha<1$. Обратное утверждение верно в силу [11, лемма 7.9$]$.

Идея второго способа доказательства теоремы 2 состоит в следующем. Хорошо известно, что эксперименты $\left(\mathrm{P}, \mathrm{P}^{\prime}\right)$ и $\left(\mathrm{Q}, \mathrm{Q}^{\prime}\right)$ эквивалентны тогда и только тогда, когда $\mathscr{L}$ aw $\left(Z_{1}^{\mathrm{P}} \mid \mathrm{P}\right)=\mathscr{L}$ aw $\left(Z_{1}^{\mathrm{Q}} \mid \mathrm{Q}\right)$, где $Z^{\mathrm{P}}$ и $Z^{\mathrm{Q}}$ - обобщенные процессы плотности меры $\mathrm{P}^{\prime}$ относительно $\mathrm{P}$ и меры $\mathrm{Q}^{\prime}$ относительно $Q$ соответственно. Поэтому достаточно показать, что распределение $\mathscr{L}$ aw $\left(Z_{1}^{\mathrm{P}} \mid \mathrm{P}\right)$ определяется, причем однозначно, через величины, стоящие в левых частях соотношений $(\mathrm{E})$.

Доказательство этого факта существенно опирается на формулу для обобщенного процесса плотности, приводимую в следующем предложении, которое является следствием более общей формулы для обобщенного процесса плотности распределений двух семимартингалов с независимыми приращениями, доказанной в статье [2]. 
Предложение 1. Пусть $\mathrm{P} \in \mathscr{P}_{L, 0}, \mathrm{P}^{\prime} \in \mathscr{P}_{I}$ u $\mathrm{P} \not \perp \mathrm{P}^{\prime}$. Тогда обобшенный процесс плотности $Z$ мерь $\mathrm{P}^{\prime}$ относительно $\mathrm{P}$ задается $\mathrm{P}-n . н$. следуюшей формулой:

$$
Z=\mathrm{P}_{0}^{\prime} \mathscr{E}(N) D=\mathrm{P}_{0}^{\prime} \mathscr{E}(N-\Psi)
$$

где

$$
\begin{aligned}
N_{t} & =\beta \cdot X_{t}^{c}+(Y-1) *(\mu-\nu)_{t}, \\
D_{t} & =\frac{\prod_{0<s \leqslant t}\left(1-a_{s}^{\prime}\right)}{\exp \left(\nu_{2}^{\prime}\left\{(s, x): 0<s \leqslant t, a_{s}^{\prime}=0\right\}\right)}, \\
\Psi_{t} & =\nu_{2}^{\prime}\left([0, t] \times \mathbf{R}^{d}\right)
\end{aligned}
$$

и $X^{c}$ - непрерывная мартингальная составляющая канонического прочесса $X$ nо мере $\mathrm{P}$.

Д ок а з а т ель с т в о т е о ре мы 2 (второй способ). Обозначим

$$
\begin{gathered}
N^{1}=-1_{\{x=-1\}} *\left(\mu^{N}-\nu^{N}\right)=-1_{\{Y=0\}} *(\mu-\nu), \\
N^{2}=N-N^{1}=\beta \cdot X_{t}^{c}+(Y-1) 1_{\{Y>0\}} *(\mu-\nu)_{t}, \\
Z^{1}=\mathscr{E}\left(N^{1}\right), \quad Z^{2}=\mathscr{E}\left(N^{2}\right),
\end{gathered}
$$

где $\mu^{N}$ и $\nu^{N}$ - соответственно мера скачков процесса $N$ и ее компенсатор (относительно Р).

Ясно, что $N^{1}$ есть чисто разрывный локальный мартингал (по мере Р) и

$$
N_{t}^{1}=-\sum_{s \leqslant t} 1_{\left\{\Delta N_{s}=-1\right\}}+\nu^{N}([0, t] \times\{-1\}) .
$$

В частности, $\Delta N^{1} \Delta N^{2}=0$ и $\left[N^{1}, N^{2}\right]=0$, поэтому из первого соотношения в (5) и формулы Йора следует, что

$$
Z^{\mathrm{P}}=\mathrm{P}_{0}^{\prime} Z^{1} Z^{2} D
$$

Дальнейшая часть доказательства опирается на лемму 4 из приложения, которая применима как к $N$, так и к $N^{1}$ (с нулевым $\beta$ и $1_{\{Y>0\}}$ в качестве $Y$ ) и $N^{2}\left(\right.$ с $Y 1_{\{Y>0\}}+1_{\{Y=0\}}$ в качестве $\left.Y\right)$. Значит, $N^{1}$ и $N^{2}$ являются локальными мартингалами с независимыми приращениями, и несложно показать, что они независимы между собой, так как $\left[N^{1}, N^{2}\right]=0$. Следовательно, $Z^{1}$ и $Z^{2}$ независимы.

Известно, что стохастическая экспонента от локального мартингала с независимыми приращениями и со скачками не менее -1 является не только локальным мартингалом, но и мартингалом (например, это вытекает из следствия $8.30[11])$, поэтому из (8) следует, что

$$
\mathrm{E}_{\mathrm{P}} Z_{1}^{\mathrm{P}}=\mathrm{P}_{0}^{\prime} D_{1}=\frac{\mathrm{P}_{0}^{\prime} \prod_{0<s \leqslant 1}\left(1-a_{s}^{\prime}\right)}{\exp \left(\nu_{2}^{\prime}\left\{(s, x): 0<s \leqslant 1, a_{s}^{\prime}=0\right\}\right)} .
$$


В силу (7) случайная величина $Z_{1}^{1}$ принимает два значения: 0 и $\exp \left\{\nu^{N}([0,1] \times\{-1\})\right\}=e^{\nu(Y=0)}$, где равенство имеет место в силу леммы 4. В силу мартингальности $Z^{1}$ имеем $\mathrm{P}\left\{Z_{1}^{1}=0\right\}=1-e^{-\nu(Y=0)}$.

В силу леммы 4 процесс $\widehat{N}=\ln Z^{2}$ есть семимартингал с независимыми приращениями без фиксированных скачков, поэтому $\widehat{N}_{1}$ имеет безгранично делимое распределение с характеристической функцией

$$
\mathrm{E} e^{i u \widehat{N}_{1}}=\exp \left\{i u B_{1}^{\widehat{N}}-\frac{1}{2} u^{2} C_{1}^{\widehat{N}}+\int\left(e^{i u x}-1-i u h_{1}(x)\right) \nu^{\widehat{N}}([0,1] \times d x)\right\}
$$

где $\left(B^{\widehat{N}}, C^{\widehat{N}}, \nu^{\widehat{N}}\right)$ - триплет локальных характеристик семимартингала $\widehat{N}$ по мере Р относительно функции усечения $h_{1}: \mathbf{R} \rightarrow \mathbf{R}$, явный вид которого указан в лемме 4. В частности,

$$
\begin{gathered}
B_{1}^{\widehat{N}}=\int_{\{Y>0\}}\left(h_{1}(\ln Y)-Y+1\right) d \nu-\frac{C_{1}^{\widehat{N}}}{2}, \\
C_{1}^{\widehat{N}}=\int_{0}^{1} \beta_{s}^{\top} c \beta_{s} d s, \\
\nu^{\widehat{N}}([0,1] \times B)=\nu(Y>0, \ln Y \in B), \quad B \in \mathscr{B}(\mathbf{R} \backslash\{0\}) .
\end{gathered}
$$

Из формул (12) и (13) следует, что величины $\int_{0}^{1} \beta_{s}^{\top} c \beta_{s} d s$ и $\nu(Y \in B)$, $B \in \mathscr{B}(\mathbf{R} \backslash\{0,1\})$, определяют $C_{1}^{\widehat{N}}$ и $\nu^{\widehat{N}}([0,1] \times B), B \in \mathscr{B}(\mathbf{R})$. То же верно относительно $B_{1}^{\widehat{N}}$, так как подынтегральная функция в (11) обращается в 0 при $Y=1$. Значит, указанные величины однозначно определяют распределения $\widehat{N}_{1}$ и $Z_{1}^{2}=e^{\widehat{N}_{1}}$ в силу (10). Как указано выше, распределение $\mathscr{L}$ aw $\left(Z_{1}^{1} \mid \mathrm{P}\right)$ полностью определяется величиной $\nu(Y=0)$. В силу независимости $Z^{1}$ и $Z^{2}$ из (8) получаем, что $\mathscr{L}$ aw $\left(Z_{1}^{\mathrm{P}} \mid \mathrm{P}\right)$ однозначно определяется величинами, входящими в левые части соотношений (Е).

Пусть, наоборот, известно $\mathscr{L}$ aw $\left(Z_{1}^{\mathrm{P}} \mid \mathrm{P}\right)$. Тогда из (9) определяется левая часть третьего соотношения в $(\mathrm{E})$.

Поскольку $\mathrm{P}_{0}^{\prime} D_{1}>0$ ввиду теоремы 4 и $Z_{1}^{2}>0$ Р-п.н. (так как $\left.\Delta N^{2}>-1\right)$, имеем $\mathrm{P}\left\{Z_{1}^{\mathrm{P}}=0\right\}=\mathrm{P}\left\{Z_{1}^{1}=0\right\}=1-e^{-\nu(Y=0)}$, что позволяет определить $\nu(Y=0)$ и $\mathscr{L}$ aw $\left(Z_{1}^{1} \mid \mathrm{P}\right)$.

Поэтому, в силу независимости $Z^{1}$ и $Z^{2}, \mathscr{L} \mathrm{aw}\left(Z_{1}^{\mathrm{P}} \mid \mathrm{P}\right)$ определяет распределение $\mathscr{L}$ aw $\left(Z_{1}^{2} \mid \mathrm{P}\right)$, а значит, и $\widehat{N}_{1}$. В силу (10) определены также $C_{1}^{\widehat{N}}$ и мера $\nu^{\widehat{N}}([0,1] \times d x)$, что ввиду $(12)$ и $(13)$ позволяет определить $\int_{0}^{1} \beta_{s}^{\top} c \beta_{s} d s$ и $\nu(Y \in B), B \in \mathscr{B}\left(\mathbf{R}_{+} \backslash\{0,1\}\right)$.

Таким образом, теорема 2 доказана.

3. Доказательство основной теоремы. Заметим, что если $\mathrm{P}^{\prime} \perp \mathrm{P}$, то эксперимент $\left(\mathrm{P}, \mathrm{P}^{\prime}\right)$ будет вполне информативным и, значит, более информативным, чем $(\mathrm{P}, \overline{\mathrm{P}})$. Поэтому, не ограничивая общности, мы полагаем ниже, что $\mathrm{P}^{\prime} \not \perp \mathrm{P}$, тогда согласно теореме 4 имеем, в частности, $C_{t}^{\prime}=c t$. Таким образом, везде ниже $C_{t}^{\prime}=c t$ и $\bar{c}=c$. 
Как отмечалось выше, схема доказательства теоремы 1 состоит в следующем: на пространстве $\mathbb{D}(\mathbf{R})$ будет построена пара мер $Q \in \mathscr{P}_{L, 0}$, $\mathrm{Q}^{\prime} \in \mathscr{P}_{L}$, для которых можно показать, что эксперимент $\left(\mathrm{Q}, \mathrm{Q}^{\prime}\right)$ эквивалентен $\left(\mathrm{P}, \mathrm{P}^{\prime}\right)$ и более информативен, чем $(\mathrm{P}, \overline{\mathrm{P}})$. Для доказательства первого из этих утверждений используется теорема 2 (см. лемму 2), а для доказательства второго - лемма о сравнении [1, лемма 5.1] (см. лемму 3$)$.

Основной шаг построения мер $\mathrm{Q}, \mathrm{Q}^{\prime}$ состоит в построении отвечающих им мер Леви $G$ и $G^{\prime}$. Основное требование на $G$ и $G^{\prime}$ состоит в том, чтобы выполнялось второе условие из (Е). Этого не всегда можно достичь, если в качестве $G$ брать $F$ (например, если $F$ сосредоточена в точке $a$, а функция $Y(t, a)$ не является постоянной п.н. по мере Лебега). Другой «естественный» кандидат на роль $G$, образ меры $\nu$ при отображении $Y$, не всегда является мерой Леви. Дополнительные требования на $G$ и $G^{\prime}$ исходят из того, что меры $\mathrm{Q}$ и $\mathrm{Q}^{\prime}$ должны быть несингулярны. Наличие сингулярной компоненты у $G^{\prime}$ по отношению к $G$ также связано с проверкой выполнения условий (Е). Одна из возможных конструкций мер $G$ и $G^{\prime}$, удовлетворяющих всем необходимым требованиям, приводится в следующей лемме.

Лемма 1. Существуют мера $G$ на $(0, \infty)$ и функиия $\varphi:(0, \infty) \rightarrow$ $\mathbf{R}_{+}$, для которых выполнены следующие условия:
a) $\int\left(x^{2} \wedge 1\right) G(d x)<\infty$
b) $\int\left(x^{2} \wedge 1\right) \varphi(x) G(d x)<\infty$,
c) $\mathscr{L}$ aw $(\varphi(x) \mid G)=\mathscr{L}$ aw $(Y(t, x) \mid \nu)$,
d) $\int(\sqrt{\varphi(x)}-1)^{2} G(d x)<\infty$,
e) $\int\left|h_{1}(x)(\varphi(x)-1)\right| G(d x)<\infty$,

где $h_{1}: \mathbf{R} \rightarrow \mathbf{R}-$ произвольная функиия усечения.

Д о к а з а т е л ь с т в о. Введем разбиение $\mathbf{R}^{d} \backslash\{0\}$ на множества

$$
\begin{aligned}
D_{1} & =\left\{x \in \mathbf{R}^{d}:|x| \geqslant 1\right\}, \\
D_{N} & =\left\{x \in \mathbf{R}^{d}: \frac{1}{N} \leqslant|x|<\frac{1}{N-1}\right\}, \quad N \geqslant 2,
\end{aligned}
$$

и разбиение $(0, \infty)$ на множества

$$
\begin{aligned}
B_{1+} & =\{x \in \mathbf{R}: x \geqslant 1\}, \\
B_{N+} & =\left\{x \in \mathbf{R}: \frac{1}{N} \leqslant x<\frac{1}{N-1}\right\}, \quad N \geqslant 2 .
\end{aligned}
$$


Зафиксируем также взаимно однозначные монотонные отображения $\psi_{N}: \mathbf{R}_{+} \rightarrow B_{N+}$.

Введем меры $G_{N}(B)$ на $\mathscr{B}\left(\mathbf{R}_{+}\right)$равенством

$$
G_{N}(B)=\nu\left((t, x) \in[0,1] \times \mathbf{R}^{d}: x \in D_{N}, Y(t, x) \in B\right), \quad N \geqslant 1,
$$

и положим

$$
G(B)=\sum_{N} G_{N} \circ \psi_{N}^{-1}(B), \quad B \in \mathscr{B}\left(\mathbf{R}_{+} \backslash\{0\}\right),
$$

где $G_{N} \circ \psi_{N}^{-1}$ - образ меры $G_{N}$ при отображении $\psi_{N}$. Определим также

$$
\varphi(x)=\sum_{N} \psi_{N}^{-1}(x) 1_{B_{N+}}(x), \quad x>0 .
$$

Условие а) выполнено, поскольку

$$
G\left(B_{N+}\right)=G_{N}\left(\mathbf{R}_{+}\right)=F\left(D_{N}\right) \leqslant N^{2} \int_{D_{N}}\left(|x|^{2} \wedge 1\right) F(d x)
$$

что дает

$$
\begin{aligned}
\int\left(x^{2} \wedge 1\right) G(d x) & =\sum_{N} \int_{B_{N+}}\left(x^{2} \wedge 1\right) G(d x) \leqslant \sum_{N} \frac{1}{(N-1)^{2} \vee 1} G\left(B_{N+}\right) \\
& \leqslant \sum_{N} \frac{N^{2}}{(N-1)^{2} \vee 1} \int_{D_{N}}\left(|x|^{2} \wedge 1\right) F(d x) \\
& \leqslant 4 \sum_{N} \int_{D_{N}}\left(|x|^{2} \wedge 1\right) F(d x)=4 \int\left(|x|^{2} \wedge 1\right) F(d x)<\infty
\end{aligned}
$$

Аналогично проверяем условие b):

$$
\begin{aligned}
\int_{\mathbf{R}_{+}} & \left(x^{2} \wedge 1\right) \varphi(x) G(d x)=\sum_{N} \int_{B_{N+}}\left(x^{2} \wedge 1\right) \varphi(x) G(d x) \\
& \leqslant \sum_{N} \frac{1}{(N-1)^{2} \vee 1} \int_{B_{N+}} \varphi(x) G(d x) \\
& =\sum_{N} \frac{1}{(N-1)^{2} \vee 1} \int_{B_{N+}} \psi_{N}^{-1}(x)\left(G_{N} \circ \psi_{N}^{-1}\right)(d x) \\
& =\sum_{N} \frac{1}{(N-1)^{2} \vee 1} \int_{\mathbf{R}_{+}} x G_{N}(d x) \\
& =\sum_{N} \frac{1}{(N-1)^{2} \vee 1} \iint_{[0,1] \times D_{N}} Y(t, x) \nu(d t, d x) \\
& \leqslant \sum_{N} \frac{N^{2}}{(N-1)^{2} \vee 1} \iint_{[0,1] \times D_{N}}\left(|x|^{2} \wedge 1\right) Y(t, x) \nu(d t, d x) \\
& \leqslant 4 \iint\left(|x|^{2} \wedge 1\right) Y(t, x) \nu(d t, d x) \\
& \leqslant 4 \iint\left(|x|^{2} \wedge 1\right) \nu^{\prime}(d t, d x)<\infty .
\end{aligned}
$$


Далее, выполнение условия с) следует из построения меры $G$, выполнение условия d) очевидно ввиду с).

Заметим также, что существует такое $N_{0} \geqslant 2$, что для произвольных функций усечения $h_{1}: \mathbf{R} \rightarrow \mathbf{R}$ и $h: \mathbf{R}^{d} \rightarrow \mathbf{R}^{d}$ при $N \geqslant N_{0}$ имеем $h_{1}(x)=x$ на $B_{N+}$ и $h(x)=x$ на $D_{N}$. Тогда для $N \geqslant N_{0}$

$$
\begin{aligned}
\int_{B_{N+}}\left|h_{1}(x)(\varphi(x)-1)\right| G(d x) & =\int_{B_{N+}}\left|x\left(\psi_{N}^{-1}(x)-1\right)\right|\left(G_{N} \circ \psi_{N}^{-1}\right)(d x) \\
& =\int_{\mathbf{R}_{+}}\left|\psi_{N}(x)(x-1)\right| G_{N}(d x) \\
& =\int_{[0,1] \times D_{N}}\left|\psi_{N}(Y(t, x))(Y(t, x)-1)\right| \nu(d t, d x) \\
& \leqslant \frac{1}{N-1} \int_{[0,1] \times D_{N}}|Y(t, x)-1| \nu(d t, d x) \\
& \leqslant \frac{N}{N-1} \int_{[0,1] \times D_{N}}|x||Y(t, x)-1| \nu(d t, d x) \\
& \leqslant 2 \int_{[0,1] \times D_{N}}|h(x)||Y(t, x)-1| \nu(d t, d x),
\end{aligned}
$$

откуда следует выполнение условия е), если воспользоваться теоремой 4 и заметить, что интеграл, стоящий в левой части предыдущей цепочки соотношений, конечен также для $N<N_{0}$ в силу а) и b). Лемма 1 доказана.

В дальнейшем всюду предполагается, что $G$ и $\varphi$ удовлетворяют условиям леммы 1 , а мера $G^{\prime}$ задается соотношением

$$
G^{\prime}(B)=\int_{B} \varphi(x) G(d x)+K \delta_{\{-1\}}(B), \quad B \in \mathscr{B}(\mathbf{R}),
$$

где $\delta_{\{-1\}}$ есть мера Дирака в точке -1 и

$$
K=\nu_{2}^{\prime}\left\{(s, x): 0<s \leqslant 1, a_{s}^{\prime}=0\right\}-\ln \prod_{t \leqslant 1: a_{t}^{\prime}>0}\left(1-a_{t}^{\prime}\right) .
$$

Конечность константы $K$ вытекает из несингулярности $\mathrm{P}$ и $\mathrm{P}^{\prime}$ (см. теорему 4), значит, $G^{\prime}$ — мера Леви ввиду условия b) леммы 1.

Введем триплеты

$$
\mathbb{T}^{\mathrm{Q}}=(0, t, d t G(d x)), \quad \mathbb{T}^{\mathbf{Q}^{\prime}}=\left(b^{\prime} t, t, d t G^{\prime}(d x)\right),
$$

где

$$
b^{\prime}=\sqrt{\int_{0}^{1} \beta_{s}^{\top} c \beta_{s} d s}+\int_{\mathbf{R}_{+}} h_{1}(x)(\varphi(x)-1) G(d x)+h_{1}(-1) K .
$$

Пусть мера $Q$ такова, что канонический процесс $X$ является процессом Леви с триплетом $\mathbb{T}^{Q}$. Определим также меру $\mathbf{Q}^{\prime}$ как меру, по 
которой $X$ является процессом с независимыми приращениями с триплетом $\mathbb{T}^{\prime}$, причем $\mathrm{Q}_{0}^{\prime}=\mathrm{P}_{0}^{\prime}$. Выполнение условий леммы 1 гарантирует существование мер $Q$ и $\mathrm{Q}^{\prime}$, а также тот факт, что $\mathrm{Q} \not \perp \mathrm{Q}^{\prime}$ (для доказательства достаточно воспользоваться теоремой 4).

Лемма 2. Экспериментьл $\left(\mathrm{P}, \mathrm{P}^{\prime}\right)$ u $\left(\mathrm{Q}, \mathrm{Q}^{\prime}\right)$ эквивалентньл.

Д о к а з а т е ль т в о. Проверим выполнение условий (Е). Из определения меры $G^{\prime}$ следует, что $Y^{\mathrm{Q}}(t, x)=\varphi(x)$, поэтому выполнено второе условие из $(\mathrm{E})$. Далее, из определения $b^{\prime}$ находим, что $\beta^{\mathrm{Q}}=\sqrt{\int_{0}^{1} \beta_{s}^{\top} c \beta_{s} d s}$, откуда следует выполнение первого условия из $(\mathrm{E})$. Наконец, имеем $\mathrm{Q}_{0}^{\prime}=\mathrm{P}_{0}^{\prime}, a_{\mathrm{Q}, t}^{\prime} \equiv 0$ и $\nu_{\mathrm{Q}, 2}^{\prime}([0,1] \times \mathbf{R})=K$, поэтому выполнение третьего условия из (Е) следует из определения константы $K$. Лемма 2 доказана.

Рассмотрим теперь эксперимент $(\mathrm{P}, \overline{\mathrm{P}})$. Нам понадобится представление для обобщенного процесса плотности $\overline{\mathrm{P}}$ относительно $\mathrm{P}$ в виде $\overline{\mathrm{P}}_{0} \mathscr{E}(\bar{N}-\bar{\Psi})$ (см. правую часть соотношения $\left.(5)\right)$ и явный вид соответствующих параметров $\bar{\beta}, \bar{Y}$, а также сингулярной компоненты меры $\bar{F}(d x)$ относительно $F(d x)$. Предварительно, конечно, следует проверить, что $\mathrm{P} \not \perp \overline{\mathrm{P}}$.

Пусть

$$
\nu_{2}^{\prime}([0,1] \times A)=\int_{A} Y_{2}(x) F(d x)+F^{\prime}(A), \quad A \in \mathscr{B}\left(\mathbf{R}^{d}\right),
$$

есть разложение меры $\nu_{2}^{\prime}([0,1] \times d x)$ на абсолютно непрерывную и сингулярную компоненты относительно $F(d x)$. Таким образом,

$$
\bar{F}(A)=\int_{A} \bar{Y}(x) F(d x)+F^{\prime}(A), \quad A \in \mathscr{B}\left(\mathbf{R}^{d}\right)
$$

где $\bar{Y}(x)=\int_{0}^{1} Y(t, x) d t+Y_{2}(x)$.

Теперь несложно проверить, что условие (ii) теоремы 4 для мер $\mathrm{P}$ и $\overline{\mathrm{P}}$ выполнено, поскольку оно выполняется для мер $\mathrm{P}$ и $\mathrm{P}^{\prime}$ (детальное рассуждение предоставляется читателю).

Пусть $g$ - такая симметрическая неотрицательно определенная матрица, что $c=g^{\top} g$. Так как $\int_{0}^{1}\left(g \beta_{s}\right)^{\top} g \beta_{s} d s=\int_{0}^{1} \beta_{s}^{\top} c \beta_{s} d s<\infty$, то интеграл $\int_{0}^{1} g \beta_{s} d s$ определен и конечен. Далее, очевидно, что существует такой вектор $\bar{\beta}$, что $g \bar{\beta}=\int_{0}^{1} g \beta_{s} d s$, и, следовательно, $c \bar{\beta}=\int_{0}^{1} c \beta_{s} d s$. Зафиксируем $\bar{\beta}$. Несложно убедиться, что для пары мер $(\mathrm{P}, \overline{\mathrm{P}})$ в разложении (18) два последних члена обращаются в нуль (т.е. выполнено условие (iv) в теореме 10), а в роли функции $\beta$ выступает функция, тождественно равная $\bar{\beta}$.

Выполнение условий (i), (iii), (vi) в теореме 4 для пары мер $(\mathrm{P}, \overline{\mathrm{P}})$ очевидно, а проверка выполнения условия (v) также предоставляется 
читателю. Таким образом,

$$
\begin{gathered}
\bar{N}=\bar{\beta} \cdot X^{c}+(\bar{Y}-1) *(\mu-\nu), \\
\bar{\Psi}_{t}=t F^{\prime}\left(\mathbf{R}^{d}\right) .
\end{gathered}
$$

Аналогичное представление для обобщенного процесса плотности $\mathrm{Q}^{\prime}$ относительно $\mathrm{Q}$ имеет вид $\mathrm{Q}_{0}^{\prime} \mathscr{E}\left(N^{\mathrm{Q}}-\Psi^{\mathrm{Q}}\right)$, где $N^{\mathrm{Q}}$ имеет представление, аналогичное (14), с коэффициентами $\beta^{\mathrm{Q}}$ и $Y^{\mathrm{Q}}$, а $\Psi_{t}^{\mathrm{Q}}=K t$.

Согласно лемме 4 из приложения, третьи характеристики процессов $\bar{N}$ и $N^{Q}$ имеют вид

$$
\nu^{\bar{N}}(d t, d x)=d t F_{\bar{N}}(d x), \quad \nu^{N} \mathrm{Q}(d t, d x)=d t F_{N} \mathrm{Q}(d x),
$$

где

$$
\begin{aligned}
F_{\bar{N}}(B) & =F\{x: \bar{Y}(x)-1 \in B\}, \quad B \in \mathscr{B}(\mathbf{R} \backslash\{0\}), \\
F_{N} \mathrm{Q}(B) & =G\{x: \varphi(x)-1 \in B\} \\
& =\nu\{(t, x): Y(t, x)-1 \in B\}, \quad B \in \mathscr{B}(\mathbf{R} \backslash\{0\}),
\end{aligned}
$$

соответственно, вторые характеристики имеют вид $C_{t}^{\bar{N}}=\bar{\beta}^{\top} c \bar{\beta} t, C_{t}^{N \mathrm{Q}}=$ $\left(\beta^{Q}\right)^{2} t$. Из однородности всех трех характеристик по времени следует, что процессы $\bar{N}$ и $N^{\mathrm{Q}}$ являются процессами Леви по мерам $\mathrm{P}$ и $\mathrm{Q}$ соответственно.

Лемма 3. Для введенных вылше мер $\mathrm{P}, \overline{\mathrm{P}}, \mathrm{Q}, \mathrm{Q}^{\prime}$ эксперимент $(\mathrm{P}, \overline{\mathrm{P}})$ менее информативен, чем $\left(\mathrm{Q}, \mathrm{Q}^{\prime}\right)$.

Д о к а з а т е ль с т в о. Заметим, что процессы плотности в начальный момент совпадают, поскольку $\overline{\mathrm{P}}_{0}=\mathrm{Q}_{0}^{\prime}$, и в силу леммы 5 достаточно рассмотреть случай $\overline{\mathrm{P}}_{0}=\mathrm{Q}_{0}^{\prime}=1$. Поэтому мы можем воспользоваться леммой о сравнении, причем, как это следует из предшествующих формулировке леммы 3 рассуждений, в ситуации, когда процессы плотности суть стохастические экспоненты от процессов Леви. В этом случае условия леммы о сравнении принимают более простой вид (см. $[1$, п. 5.4, условия (5.32)-(5.34)]). А именно, утверждение леммы 3 будет доказано, если проверить следующие условия (15)-(17):

$$
\bar{\beta}^{\top} c \bar{\beta} \leqslant\left(\beta^{\mathrm{Q}}\right)^{2},
$$

для каждого $w \in(-1,0)$

$$
\int(w-x)^{+} F_{\bar{N}}(d x) \leqslant \int(w-x)^{+} F_{N} \mathrm{Q}(d x),
$$

для каждого $w \in(0, \infty)$

$$
\int(x-w)^{+} F_{\bar{N}}(d x)+F^{\prime}\left(\mathbf{R}^{d}\right) \leqslant \int(x-w)^{+} F_{N} \mathbf{Q}(d x)+K .
$$


Условие (15) выполнено, так как

$$
\begin{aligned}
\bar{\beta}^{\top} c \bar{\beta} & =(g \bar{\beta})^{\top}(g \bar{\beta})=\left(\int_{0}^{1} g \beta_{s} d s\right)^{\top}\left(\int_{0}^{1} g \beta_{s} d s\right) \\
& \leqslant \int_{0}^{1}\left(g \beta_{s}\right)^{\top}\left(g \beta_{s}\right) d s=\int_{0}^{1} \beta_{s}^{\top} c \beta_{s} d s=\left(\beta^{\mathrm{Q}}\right)^{2} .
\end{aligned}
$$

Далее, для $w \in(-1,0)$

$$
\begin{aligned}
\int(w & -x)^{+} F_{\bar{N}}(d x)=\int(1+w-\bar{Y}(x))^{+} F(d x) \\
& \leqslant \int\left(1+w-\int Y(t, x) d t\right)^{+} F(d x) \leqslant \iint(1+w-Y(t, x))^{+} d t F(d x) \\
& =\int(w-x)^{+} F_{N} \mathrm{Q}(d x),
\end{aligned}
$$

т.е. условие (16) выполнено.

Наконец, для $w \in(0, \infty)$

$$
\begin{aligned}
& \int(x-w)^{+} F_{\bar{N}}(d x)+F^{\prime}\left(\mathbf{R}^{d}\right)=\int(\bar{Y}(x)-1-w)^{+} F(d x)+F^{\prime}\left(\mathbf{R}^{d}\right) \\
& \leqslant \int\left(\int Y(t, x) d t-1-w\right)^{+} F(d x)+\int Y_{2}(x) F(d x)+F^{\prime}\left(\mathbf{R}^{d}\right) \\
& \leqslant \iint(Y(t, x)-1-w)^{+} d t F(d x)+\nu_{2}^{\prime}\left([0,1] \times \mathbf{R}^{d}\right) \\
& \leqslant \int(x-w)^{+} F_{N} \mathrm{Q}(d x)+K,
\end{aligned}
$$

где последнее неравенство в цепочке следует из того, что

$$
\begin{aligned}
& \nu_{2}^{\prime}\left([0,1] \times \mathbf{R}^{d}\right)=\nu_{2}^{\prime}\left\{(s, x): 0<s \leqslant 1, a_{s}^{\prime}=0\right\}+\sum_{t \leqslant 1} a_{t}^{\prime} \\
& \quad \leqslant \nu_{2}^{\prime}\left\{(s, x): 0<s \leqslant 1, a_{s}^{\prime}=0\right\}-\ln \prod_{t \leqslant 1: a_{t}^{\prime}>0}\left(1-a_{t}^{\prime}\right)=K .
\end{aligned}
$$

Таким образом, условие (17) также выполнено. Лемма 3 доказана.

У тверждение теоремы 1 есть непосредственное следствие лемм 2 и 3.

4. Применение к одной задаче минимизации $f$-дивергенции. Укажем на некоторую связь данной работы с результатами ряда работ (среди которых наиболее близкими являются [8], [10] и [14]), мотивированных приложениями к финансовой математике.

Пусть $S-d$-мерный семимартингал на стохастическом базисе $(\Omega, \mathscr{F}, \mathbb{F}, Q)$, интерпретируемый как дисконтированный процесс цен $d$ активов. Пусть $\mathscr{M}^{\text {a }}(S)$ - множество вероятностных мер $\widetilde{Q}$, абсолютно непрерывных относительно $\mathrm{Q}$ и таких, что $S$ - локальный мартингал 
относительно $\widetilde{Q}$. В задаче максимизации полезности стандартный подход состоит в том, что сначала решается двойственная задача, заключающаяся в минимизации $f$-дивергенции $\mathscr{J}_{f}(\widetilde{\mathrm{Q}}, \mathrm{Q})$ по $\widetilde{\mathrm{Q}} \in \mathscr{M}^{\mathrm{a}}(S)$ для некоторых $f \in \mathscr{C}$ (см., например, [16] и [20]).

Весьма распространенное предположение о модели финансового рынка состоит в том, что процесс цен $S$ есть экспонента от процесса Леви (см., например, [5], [7], [8]-[10], [13]-[15]). В силу [12, теорема II.8.10] это эквивалентно тому, что $S=\mathscr{E}(L)$, где $L-$ процесс Леви с $\Delta L>-1$. Заметим, что $S$ является локальным мартингалом по мере $\widetilde{Q}$ тогда и только тогда, когда $L$ является локальным мартингалом по мере $\widetilde{\mathrm{Q}}$, т.е. $\mathscr{M}^{\mathrm{a}}(S)=\mathscr{M}^{\mathrm{a}}(L)$.

Поэтому естественно рассмотреть следующую задачу. Пусть $L-$ процесс Леви относительно меры $Q$ на некотором стохастическом базисе. Пусть $\mathscr{M}^{\mathrm{a}}(L)$ - множество вероятностных мер $\widetilde{\mathrm{Q}}$, абсолютно непрерывных относительно $Q$ и таких, что $L$ - локальный мартингал относительно $\widetilde{Q}$. Требуется минимизировать $f$-дивергенцию $\mathscr{J}_{f}(\widetilde{Q}, Q)$ по $\widetilde{\mathrm{Q}} \in \mathscr{M}^{\mathrm{a}}(L)$.

Для некоторых выпуклых функций $f$ при тех или иных предположениях было доказано, что если эта задача имеет решение, то экстремум достигается на мере, по которой $L$ является процессом Леви, а именно, случай $f(x)=x \ln x$ рассматривается в [8], случай $f(x)=x^{q}$ для $q \in(-\infty, 0) \cup(1, \infty)$ — в [14], а из результатов работы [10] несложно вывести условия, при которых такой результат справедлив для $f(x)=-\ln x$. Для произвольной выпуклой функции $f$, по-видимому, среди экстремальных точек не обязательно найдется мартингальная мера, по которой $L$ является процессом Леви (см., например, [4]).

Однако следующий результат показывает, что если экстремум (конечный) достигается на мере $\mathrm{Q}^{\prime} \in \mathscr{M}^{\mathrm{a}}(L)$, относительно которой $L$ есть процесс с независимыми приращениями, то экстремум будет также достигаться и на некоторой мере $\overline{\mathrm{Q}} \in \mathscr{M}^{\mathrm{a}}(L)$, по которой $L$ есть процесс Леви (меры $\bar{Q}$ и $\mathrm{Q}^{\prime}$ не обязаны совпадать, так как если $f$ не строго выпукла, то экстремум может достигаться в разных точках).

Теорема 3. Пусть на произвольном измеримом пространстве с $\oint$ фльтрацией $(\Omega, \mathscr{F}, \mathbb{F})$ заданьл $d$-мерньии проиесс $L$ на интервале $[0,1] c$ непрерьвными справа и имеюшими предель слева траекториями, мера $\mathrm{Q}$, относительно которой $L$ является прочессом Леви, и мера $\mathrm{Q}^{\prime} \ll \mathrm{Q}$, относительно которой $L$ является мартингалом с независимьми приращениями. Тогда на том же пространстве найдется мера $\overline{\mathrm{Q}}$ такая, что

1) $\bar{Q} \ll Q$,

2) $L$ - мартингал и процесс Леви относительно мерь $\bar{Q}$,

3) $J_{f}(\overline{\mathrm{Q}}, \mathrm{Q}) \leqslant J_{f}\left(\mathrm{Q}^{\prime}, \mathrm{Q}\right)$ для всех $f \in \mathscr{C}$.

всли $\mathrm{Q}^{\prime} \sim \mathrm{Q}$, mо $\overline{\mathrm{Q}} \sim \mathrm{Q}$. 
Д о к а з а т е л ь с т в о. Обозначим $(B, C, \nu)$ детерминированную и однородную по времени версию триплета $L$ относительно меры Q (см., например, [12, следствие II.4.19]), где

$$
B_{t}(\omega)=b t, \quad C_{t}(\omega)=c t, \quad \nu(\omega ; d t, d x)=d t F(d x) .
$$

Обозначим $\left(B^{\prime}, C^{\prime}, \nu^{\prime}\right)$ детерминированную версию триплета $L$ относительно меры $\mathrm{Q}^{\prime}$.

Пусть $\mathrm{P}$ и $\mathrm{P}^{\prime}$ — меры на пространстве Скорохода, отвечающие распределениям $L$ по мерам $\mathrm{Q}$ и $\mathrm{Q}^{\prime}$ соответственно, тогда триплеты канонического процесса $X$ относительно $\mathrm{P}$ и $\mathrm{P}^{\prime}$ будут соответственно $(B, C, \nu)$ и $\left(B^{\prime}, C^{\prime}, \nu^{\prime}\right)$ в силу того, что триплеты процессов с независимыми приращениями однозначно определяются по их распределениям (см., например, [12, теоремы II.4.15, II.5.2 и следствие II.5.11]). Очевидно, что из $\mathrm{Q}^{\prime} \ll \mathrm{Q}$ следует, что $\mathrm{P}^{\prime} \ll \mathrm{P}$, в частности, $C_{t}^{\prime}=c t$ и $X_{0}=0$ Р- и $\mathrm{P}^{\prime}$-п.н.

По распределению $\mathrm{P}^{\prime}$ построим распределение $\overline{\mathrm{P}} \in \mathscr{P}_{L}$ как в п. 1. Отметим, что $\overline{\mathrm{P}} \in \mathscr{P}_{L, 0}$ в силу совпадения начальных распределений процесса $X$ по мерам $\mathrm{P}^{\prime}$ и $\overline{\mathrm{P}}$.

Далее, абсолютная непрерывность сохраняется при переходе к менее информативному эксперименту, поэтому $\overline{\mathrm{P}} \ll \mathrm{P}$ и $\overline{\mathrm{P}} \sim \mathrm{P}$, если $\mathrm{Q}^{\prime} \sim \mathrm{Q}$ и, значит, $\mathrm{P}^{\prime} \sim \mathrm{P}$.

Поскольку $\mathrm{P}, \overline{\mathrm{P}} \in \mathscr{P}_{L, 0}$, процесс плотности $\overline{\mathrm{P}}$ относительно $\mathrm{P}$ имеет вид $\mathscr{E}\left(N^{X}\right)$ с $N^{X}=\beta \cdot X^{c}+(Y-1) *\left(\mu^{X}-\nu^{X}\right)$, где параметры Гирсанова $\beta, Y$ не зависят ни от случая, ни от времени; здесь $\nu^{X}=\nu$ есть Ркомпенсатор меры скачков $\mu^{X}$ канонического процесса $X$. В частности, $\beta \in L_{\text {loc }}^{2}\left(X^{c}, \mathrm{P}\right)$ и $(Y-1) \in G_{\mathrm{loc}}\left(\mu^{X}, \mathrm{P}\right)$. Поскольку триплеты процесса $X$ относительно $\mathrm{P}$ и процесса $L$ относительно $\mathrm{Q}$ совпадают и детерминированы, то, учитывая [12, теорема II.1.33], мы получим, что корректно определен процесс $Z=\mathscr{E}\left(N^{L}\right)$ с $N^{L}=\beta \cdot L^{c}+(Y-1) *\left(\mu^{L}-\nu^{L}\right)$, где $\nu^{L}=\nu$ есть Q-компенсатор меры скачков $\mu^{L}$ процесса $L$.

По лемме 4 (см. п. 5 ниже) триплеты процессов $N^{X}$ и $N^{L}$ относительно мер $\mathrm{P}$ и $\mathrm{Q}$ соответственно совпадают. Отсюда легко следует, что распределения стохастических экспонент $\mathscr{E}\left(N^{X}\right)$ и $\mathscr{E}\left(N^{L}\right)$ по мерам Р и $\mathrm{Q}$ соответственно совпадают. Действительно, если $Y>0 F$-п.в., это следует из [12, теорема II.8.10]. В общем случае применим тот же прием с выделением скачков процессов $N^{X}$ и $N^{L}$, равных -1 , как при втором способе доказательства теоремы 3.

Далее, процесс $Z$ является неотрицательным локальным мартингалом, а в силу [11, следствие 8.30] — мартингалом со средним 1. Таким образом, $Z$ есть процесс плотности некоторой вероятностной меры $\bar{Q}$ относительно $Q$, и из доказанного в предыдущем абзаце следует, что эксперименты $(P, \bar{P})$ и $(Q, \bar{Q})$ эквивалентны.

По теореме Гирсанова для семимартингалов (см. теорему III.3.24 в [12]) семимартингал $L$ по мере $\bar{Q}$ имеет те же характеристики, что и 
$X$ относительно меры $\overline{\mathrm{P}}$, в частности, $L$ есть процесс Леви по мере $\overline{\mathrm{Q}}$. В силу того, что свойство семимартингала быть локальным мартингалом выражается в терминах триплетов его характеристик, оно сохраняется при переходе от $\mathrm{Q}^{\prime}$ к $\mathrm{P}^{\prime}$ и от $\overline{\mathrm{P}}$ к $\overline{\mathrm{Q}}$, а в п. 1 доказано, что оно сохраняется и при переходе от $\mathrm{P}^{\prime}$ к $\overline{\mathrm{P}}$, поэтому $L$ есть локальный мартингал, а значит, и мартингал по мере $\overline{\mathbf{Q}}$.

Наконец,

$$
\mathscr{J}_{f}(\overline{\mathrm{Q}}, \mathrm{Q})=\mathscr{J}_{f}(\overline{\mathrm{P}}, \mathrm{P}) \leqslant \mathscr{J}_{f}\left(\mathrm{P}^{\prime}, \mathrm{P}\right) \leqslant \mathscr{J}_{f}\left(\mathrm{Q}^{\prime}, \mathrm{Q}\right),
$$

где первое равенство следует из эквивалентности экспериментов $(Q, \bar{Q})$ и $(\mathrm{P}, \overline{\mathrm{P}})$, второе неравенство есть следствие теоремы 1 , а третье неравенство - следствие рандомизационного критерия (см., например, [17, следствие 4.17]). Теорема 3 доказана.

5. Приложение: вспомогательные результаты. Пусть, как и в п. $2, \mathrm{P} \in \mathscr{P}_{L, 0}, \mathrm{P}^{\prime} \in \mathscr{P}_{I}$. Для удобства читателя мы сформулируем критерий отсутствия сингулярности $\mathrm{P}$ и $\mathrm{P}^{\prime}$, неоднократно используемый в работе. Введем еще несколько объектов, помимо введенных в п. 2.

Обозначим $\mathrm{P}_{H}, \mathrm{P}_{H}^{\prime}$ распределения $X_{0}$ по мерам $\mathrm{P}, \mathrm{P}^{\prime}$ соответственно. Далее, существуют непрерывная возрастающая функция $A_{t}$ и две функции $c_{t}, c_{t}^{\prime}$ со значениями в множестве симметрических неотрицательно определенных матриц размера $d \times d$ такие, что $C=c \cdot A, C^{\prime}=c^{\prime} \cdot A$.

Положим $\Sigma=\left\{t:\left|h(x)\left(U-U^{\prime}\right)\right| * \lambda_{t}<\infty\right\}$. На множестве $\Sigma$ существует разложение

$$
B^{\prime}=B+h(x)\left(U^{\prime}-U\right) * \lambda+(c \beta) \cdot A+\beta^{\perp} \cdot A+\widetilde{B},
$$

где $\beta$ и $\beta^{\perp}$ - измеримые функции из $\Sigma$ в $\mathbf{R}^{d}, \widetilde{B}-$ функция из $\Sigma$ в $\mathbf{R}^{d}$ с ограниченной вариацией на компактных интервалах, $d \widetilde{B}$ и $d A$ взаимно сингулярны на $\Sigma$ и для всех $t$ вектор $\beta_{t}^{\perp}$ ортогонален образу $\mathbf{R}^{d}$ при линейном отображении, связанном с матрицей $c_{t}$.

Введем также

$$
h^{0}\left(\frac{1}{2}\right)=\frac{1}{8}\left(\beta^{\top} c \beta 1_{\Sigma}\right) \cdot A+\frac{1}{2}\left(\sqrt{U}-\sqrt{U^{\prime}}\right)^{2} * \lambda+\frac{1}{2} \sum_{s \leqslant \cdot}\left(1-\sqrt{1-a_{s}^{\prime}}\right)^{2} .
$$

Tеорема 4 (критерий отсутствия сингулярности, теорема IV.4.33 из [12]). Для того чтобь $\mathrm{P}^{\prime}$ и $\mathrm{P}$ были несингулярны, необходимо и достаточно, чтобь выполнялись следующие условия:
(i) $P_{H}^{\prime}$ и $P_{H}$ несингулярнь;
(ii) $\left|h(x)\left(U-U^{\prime}\right)\right| * \lambda_{1}<\infty$;
(iii) $C_{t}=C_{t}^{\prime} \partial \Omega я$ всех $t \in[0,1]$;
(iv) $\beta^{\perp} \cdot A_{t}+\widetilde{B}_{t}=0$ длs всеx $t \in[0,1]$;
(v) $h^{0}(1 / 2)_{1}<\infty$;
(vi) $a_{t}^{\prime}<1$ для всеx $t \in[0,1]$. 
В следующей вспомогательной лемме вычисляются характеристики процесса, представимого в виде суммы стохастических интегралов по непрерывной мартингальной составляющей и скомпенсированной мере скачков процесса Леви с детерминированными подынтегральными функциями. Предполагается, что $X=\left(X_{t}\right)_{t \in[0,1]}$ есть $d$-мерный случайный процесс на стохастическом базисе $\left(\Omega, \mathscr{F},\left(\mathscr{F}_{t}\right), \mathrm{P}\right)$.

Предполагается также, что первая характеристика триплета процесса $X$ отвечает функции усечения $h: \mathbf{R}^{d} \rightarrow \mathbf{R}^{d}$, а первые характеристики триплетов вводимых в лемме 4 процессов $N$ и $\widehat{N}$ отвечают функции усечения $h_{1}: \mathbf{R} \rightarrow \mathbf{R}$.

Лемма 4. Пусть $X$ есть $d$-мерньий прочесс Леви относительно мерь Р с характеристиками (bt,ct, $d t F(d x)), X^{c}$ - непрерьвная мартингальная составляющая $X$ и $\mu^{X}$ - его мера скачков. Обозначим $\nu^{X}=d t F(d x)$. Пусть задань также функиии $\beta:[0,1] \rightarrow \mathbf{R}^{d} u$ $Y:[0,1] \times \mathbf{R}^{d} \rightarrow \mathbf{R}_{+}$, причем $\beta \in L_{\mathrm{loc}}^{2}\left(X^{c}\right), Y-1 \in G_{\mathrm{loc}}\left(\mu^{X}\right)$ (определения соответствуюших классов см. в [12, nn. III.4.3, II.1.27]).

Тогда прочесс $N=\beta \cdot X^{c}+(Y-1) *\left(\mu^{X}-\nu^{X}\right)$ является мартингалом с независимыми приращениями и версия его характеристик $\left(B^{N}, C^{N}, \nu^{N}\right)$ задается соотношениями

$$
\begin{gathered}
B^{N}=\left(h_{1}(x)-x\right) * \nu^{N}, \quad C_{t}^{N}=\int_{0}^{1} \beta_{s}^{\top} c \beta_{s} d s, \\
\nu^{N}(A)=\nu^{X}\{(t, x): Y(t, x) \neq 1,(t, Y(t, x)-1) \in A\}, \quad A \in \mathscr{B}([0,1] \times \mathbf{R}) .
\end{gathered}
$$

Пусть дополнительно $Y>0$ всюду, тогда процесс $\widehat{N}=\ln \mathscr{E}(N)$ корректно определен, является семимартингалом с независимьми приращениями и его характеристики $\left(B^{\widehat{N}}, C^{\widehat{N}}, \nu^{\widehat{N}}\right)$ задаются соотношениями

$$
\begin{gathered}
B^{\widehat{N}}=\left(h_{1}(\ln (1+x))-x\right) * \nu^{N}-\frac{C^{\widehat{N}}}{2}, \quad C_{t}^{\widehat{N}}=\int_{0}^{1} \beta_{s}^{\top} c \beta_{s} d s, \\
\nu^{\widehat{N}}(A)=\nu^{X}\{(t, x): Y(t, x) \neq 1,(t, \ln Y(t, x)) \in A\}, \quad A \in \mathscr{B}([0,1] \times \mathbf{R}) .
\end{gathered}
$$

Д о к а з а т е л ь с т в о. Процесс $N$ является локальным мартингалом в силу свойств стохастических интегралов; из этих свойств также следует, что

$$
C_{t}^{N}=\left\langle N^{c}, N^{c}\right\rangle_{t}=\int_{0}^{1} \beta_{s}^{\top} c \beta_{s} d s
$$

Далее, пусть $A \in \mathscr{B}([0,1] \times \mathbf{R}), \widetilde{A}=\left\{(t, x) \in[0,1] \times \mathbf{R}^{d}: Y(t, x) \neq\right.$ $1,(t, Y(t, x)-1) \in A\}$. Поскольку $\Delta N_{t}=\left(Y\left(t, \Delta X_{t}\right)-1\right) 1_{\left\{\Delta X_{t} \neq 0\right\}}$, имеем $1_{A} * \mu^{N}=1_{\tilde{A}} * \mu^{X}$.

Предположим дополнительно, что $A \subseteq[0,1] \times\{x:|x|>\varepsilon\}$ для некоторого $\varepsilon>0$. Тогда процесс $1_{A} * \mu^{N}$ локально интегрируем и его 
компенсатором является $1_{A} * \nu^{N}$, который, таким образом, совпадает с компенсатором процесса $1_{\tilde{A}} * \mu^{X}$, равным $1_{\tilde{A}} * \nu^{X}$. Это доказывает формулу для $\nu^{N}(A)$ при $A \subseteq[0,1] \times\{x:|x|>\varepsilon\}$, что, очевидно, достаточно, так как $\nu^{N}$ не нагружает множество $[0,1] \times\{0\}$ и $\widetilde{A}=\varnothing$, если $A \subseteq[0,1] \times\{0\}$

Выражение для первой характеристики $B^{N}$ получим из того, что $N$ является локальным мартингалом (см. [12, предложение II.2.29]).

Если функция $Y$ строго положительна, имеем $\Delta N>-1$ и $\mathscr{E}(N)>0$, $\mathscr{E}(N)_{-}>0$ Р-п.н., поэтому процесс $\widehat{N}=\ln \mathscr{E}(N)$ корректно определен и является семимартингалом. Более того, его характеристики можно вычислить через характеристики $N$ с помощью теоремы II.8.10 из [12]. Формулу для $\nu^{\widehat{N}}$ можно также получить аналогично формуле для $\nu^{N} \mathrm{c}$ учетом того, что $\Delta \widehat{N}_{t}=\ln (1+\Delta N)=\left[\ln Y\left(t, \Delta X_{t}\right)\right] 1_{\left\{\Delta X_{t} \neq 0\right\}}$.

Наконец, из того, что построенные версии триплетов для $N$ и $\widehat{N}$ являются детерминированными, следует, что $N$ и $\widehat{N}$ являются процессами с независимыми приращениями, а из [11, теорема 5.16] следует, что $N$ - мартингал. Лемма 4 доказана.

Следующее вспомогательное утверждение используется в доказательстве леммы 3 в п. 3 .

Лемма 5. Пусть для некоторого $0<c<1$ обобщеннье плотности экспериментов $(\widehat{\mathrm{P}}, \mathrm{P}),(\overline{\mathrm{P}}, \mathrm{P}),(\widehat{\mathrm{Q}}, \mathrm{Q}),(\overline{\mathrm{Q}}, \mathrm{Q})$ связань следующими соотношениями:

$$
\frac{d \widehat{\mathrm{P}}}{d \mathrm{P}}=Z^{\mathrm{P}}, \quad \frac{d \overline{\mathrm{P}}}{d \mathrm{P}}=c Z^{\mathrm{P}}, \quad \frac{d \widehat{\mathrm{Q}}}{d \mathrm{Q}}=Z^{\mathrm{Q}}, \quad \frac{d \overline{\mathrm{Q}}}{d \mathrm{Q}}=c Z^{\mathrm{Q}} .
$$

Соотношение $(\widehat{\mathrm{P}}, \mathrm{P}) \succcurlyeq(\widehat{\mathrm{Q}}, \mathrm{Q})$ справедливо тогда и только тогда, когда $(\overline{\mathrm{P}}, \mathrm{P}) \succcurlyeq(\overline{\mathrm{Q}}, \mathrm{Q})$.

Д о к а з а т ель с т в о. Поставим в соответствие каждому $\pi \in$ $[0,1]$ число $\widehat{\pi}=c \pi /(1-\pi+c \pi)$. Очевидно, что это взаимно однозначное отображение отрезка $[0,1]$ на себя и $f_{\hat{\pi}}(x)=f_{\pi}(c x) /(1-\pi+c \pi)$, где $f_{\pi}(x)=\max \{1-\pi-\pi x, 0\}, \pi \in[0,1]$. Далее, имеем

$$
\begin{gathered}
J_{f_{\pi}}(\overline{\mathrm{P}}, \mathrm{P})=\mathrm{E}_{\mathrm{P}} f_{\pi}\left(c Z^{\mathrm{P}}\right), \quad J_{f_{\hat{\pi}}}(\widehat{\mathrm{P}}, \mathrm{P})=\mathrm{E}_{\mathrm{P}} f_{\hat{\pi}}\left(Z^{\mathrm{P}}\right)=\frac{\mathrm{E}_{\mathrm{P}} f_{\pi}\left(c Z^{\mathrm{P}}\right)}{1-\pi+c \pi} \\
J_{f_{\pi}}(\overline{\mathrm{Q}}, \mathrm{Q})=\mathrm{E}_{\mathrm{Q}} f_{\pi}\left(c Z^{\mathrm{P}}\right), \quad J_{f_{\hat{\pi}}}(\widehat{\mathrm{Q}}, \mathrm{Q})=\mathrm{E}_{\mathrm{Q}} f_{\hat{\pi}}\left(Z^{\mathrm{Q}}\right)=\frac{\mathrm{E}_{\mathrm{Q}} f_{\pi}\left(c Z^{\mathrm{Q}}\right)}{1-\pi+c \pi}
\end{gathered}
$$

откуда с использованием соотношения для минимального байесовского риска $b_{(\overline{\mathrm{P}}, \mathrm{P})}(\pi)=1-\pi-J_{f_{\pi}}(\overline{\mathrm{P}}, \mathrm{P})$ и одного из эквивалентных определений сравнимости экспериментов через сравнимость минимальных байесовских рисков (см., например, [1, предложение 2.1]) следует утверждение леммы. 


\section{СПИСОК ЛИТЕРАТУРЫ}

1. Гущин А. А., Мордецки Э. Границы цен опционов для семимартингальных моделей рынка. - Тр. МИАН, 2002, т. 237, с. 80-122.

2. Хихол С. А. Формула для обобщенного процесса плотности распределений семимартингалов с независимыми приращениями. - Теория вероятн. и ее примен., 2009, т. 54 , в. 4, с. $716-729$.

3. Ширяев A.Н., Черный A.C. Векторный стохастический интеграл и фундаментальные теоремы теории арбитража. - Тр. МИАН, 2002, т. 237, с. 12-56.

4. Cawston S., Vostrikova L. An f-divergence approach for optimal portfolios in exponential Lévy models. arXiv:1012.3136 12/2010.

5. Chan T. Pricing contingent claims on stocks driven by Lévy processes. - Ann. Appl. Probab., 1999, v. 9, № 2, p. 504-528.

6. Coquet F., Jacod J. Convergence des surmartingales. Application aux vraisemblances partielles. - Lecture Notes in Math., 1990, v. 24, p. 282-299.

7. Eberlein E., Jacod J. On the range of options prices. - Finance Stoch., 1997, v. 1, № 2, p. 131-140.

8. Esche F., Schweizer M. Minimal entropy preserves the Lévy property: how and why. - Stochastic Process. Appl., 2005, v. 115, № 2, p. 299-327.

9. Hubalek F., Sgarra C. Esscher transforms and the minimal entropy martingale measure for exponential Lévy models. - Quant. Finance, 2006, v. 6, № 2, p. 125-145.

10. Hurd T. R. A note on log-optimal portfolios in exponential Lévy markets. - Statist. Decisions, 2004, v. 22, № 3, p. 225-233.

11. Jacod J. Calcul Stochastique et Problèmes de Martingales. — Lecture Notes in Math., 1979 , v. 714 , p. $1-539$.

12. Jacod J., Shiryaev A. N. Limit Theorems for Stochastic Processes. Berlin: SpringerVerlag, 2003, 661 p. (Grundlehren Math. Wiss., v. 228.)

13. Jakubenas P. On option pricing in certain incomplete markets. - Тр. МИАН, 2002, т. 237 , c. $123-142$.

14. Jeanblanc M., Klöppel S., Miyahara $Y$. Minimal $f^{q}$-martingale measures of exponential Lévy processes. - Ann. Appl. Probab., 2007, v. 17, № 5-6, p. 1615-1638.

15. Kallsen J. Optimal portfolios for exponential Lévy processes. - Math. Methods Oper. Res., 2000, v. 51, № 3, p. 357-374.

16. Kramkov D., Schachermayer $W$. The asymptotic elasticity of utility functions and optimal investment in incomplete markets. - Ann. Appl. Probab., 1999, v. 9, № 3, p. 904-950.

17. Liese F., Miescke K.-J. Statistical Decision Theory. New York: Springer, 2008.

18. Liese F., Vajda I. Convex Statistical Distances. Leipzig: Teubner, 1987, 224 p.

19. Mémin J., Shiryayev A. N. Distance de Hellinger-Kakutani des lois correspondant à deux processus à accroissements indépendants. - Z . Wahrscheinlichkeitstheor. verw. Geb., 1985, v. 70, № 1, p. 67-89.

20. Schachermayer $W$. Optimal investment in incomplete markets when wealth may become negative. - Ann. Appl. Probab., 2001, v. 11, № 3, p. 694-734.

21. Shiryaev A.N., Spokoiny V. G. Statistical Experiments and Decisions. Asymptotic Theory. River Edge: World Scientific Publishing Co., 2000, 281 p.

22. Strasser H. Mathematical Theory of Statistics. Berlin: de Gruyter, 1985, 492 p.

23. Torgersen E. Comparison of Statistical Experiments. Cambridge: Cambridge Univ. Press, 1991, 675 p.

Поступила в редакцию

3.V.2011

Исправленный вариант

29.VI.2011 